\title{
Evaluation of the Performance of Telecommunication Systems by Approach of Hybrid Stochastic Automata Combined With Neuro-Fuzzy Networks
}

\author{
Offole Florence (Assistant) \\ Atangana Ateba (Professor) \\ Department of Mechanics and Productics, University of Douala, Cameroon \\ Kombe Timothée (PhD) \\ Department of Industrial maintenance, University of Douala, Cameroon
}

Fohoue Kennedy (Assistant)

Department Electronics, Electrotechnics and Automation,

University of Ngaoundere, Cameroon

doi: 10.19044/esj.2017.v13n18p498 URL:http://dx.doi.org/10.19044/esj.2017.v13n18p498

\begin{abstract}
This paper presents a functional and dysfunctional behavioral study of a telecommunication system, with the aim to evaluate the performance of its constituent units. It is question of taking advantage offered by artificial intelligence in order to evaluate by modeling and simulation in system reliability. The methodological approach consists in combining ANFIS neuro-fuzzy networks with hybrid stochastic automata. The Neuro-Fuzzy ANFIS networks provide a prediction for the passage from nominal mode to degraded mode, by controlling the occurrence of malfunctions at transient levels. This allows to anticipate the occurrence of events degrading system performance, such as failures and disturbances. The objective is to maintain the system in nominal operating mode and prevent its tipping in degraded mode. The results are implanted around a demonstrator based on Scilab, and implemented on Matlab / Simulink.
\end{abstract}

Keywords: Performance Evaluation, Modeling, Reliability, Telecommunication systems, Neuro-Fuzzy networks, hybrid stochastic automata

\section{Introduction}

Performance evaluation is a difficult problem because it requires taking into account the different constituent parts of a system (human, organizational, technical), participating in a way differentiated in its global 
performance (Kombe, 2009).

With the increasing complexity the systems of communication and the importance which we attached to their capacity to work correctly and in a continuous way, the need to model accurately their functional and dysfunctional behavior and then to estimate their global performance is more and more pressing. Various performance indicators and modeling tools are already commonly used in the field of operating safety of the telecommunication systems. We can quote the reliability (Villemeur, 1988), an important parameter of operating safety because the network is regularly submitted to interferences, responsible for instability and fluctuations in the covered zone (Ruckus, 2009). Other parameters of the operating safety are: Maintainability, Availability and Safety. There is also the problem of access control operated by the operator, and the protection of the exchanges of data between knots (state) (Ben, 2007) and (Lupan, 2006).

The mathematical complexity of the evaluation of the operating safety of the dynamic systems leads us to resort to simulation. The structure of the dynamic system chosen is the Hybrid Stochastic Automaton. It is hybrid because it has two states namely: continuous state (which is the flow of message) and discrete state (which are operating states of various knots), that are defined by systems. The use of a Neuro-fuzzy network in the module monitoring/control offers the possibility to model prior to knowledge and linguistic rules of decisions obtained by experts in the field. It takes advantage of the capacities of the fuzzy inference modelled by a parallel architecture (Benaicha, 2013) and (Houacine, 2016). Hence the interest of combining Hybrid Stochastic Automata with Neuro-Fuzzy Networks.

\section{Detection and diagnosis in telecommunication system}

To detect failures in a telecommunication system, one must be able to classify observable situations as being normal (functioning) or abnormal (degraded) (Lefevre, 2000). This classification is not trivial, given the lack of information that generally characterizes abnormal situations. A commonly adopted simplification consists in considering as abnormal any situation which is not normal. 


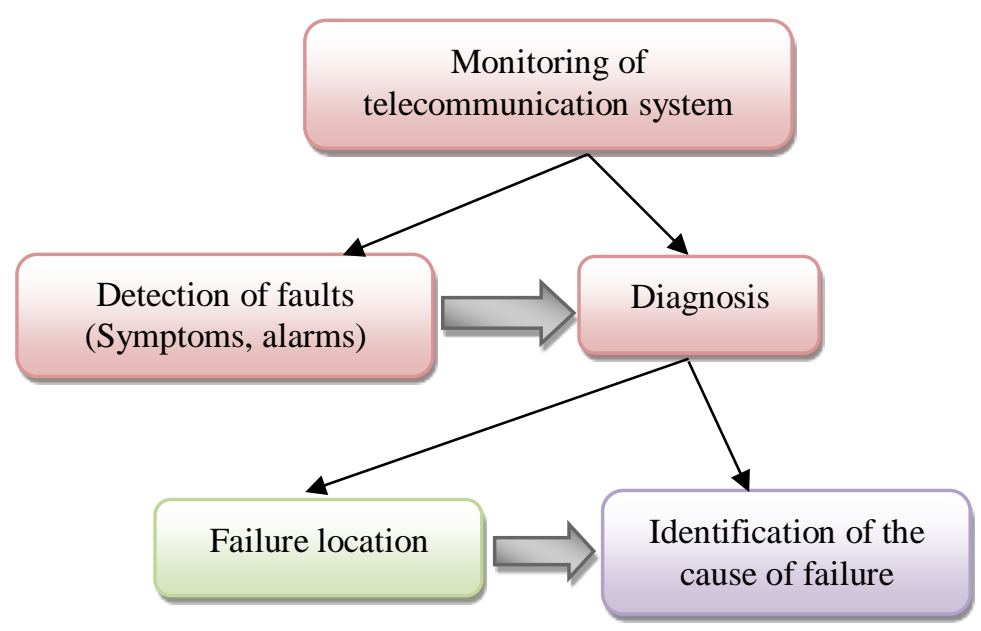

Fig. 1: Components of telecommunication system monitoring

Given a set of observed demonstrations (symptoms, observation, etc.) (Fig. 1), it is question of explaining their presence, going back to the causes, using knowledge about the telecommunication system. Furthermore, this concept also applies to the decomposition of the diagnosis into two functions : localization, which makes it possible to determine the failing functional subset and the identification, which must determine the causes that led to an abnormal situation. Finally, in this definition, the diagnosis is made from observations, what means well considering the detection as not taking part in the phase of diagnosis.

Two types of knowledge data are considered necessary for the diagnosis operation :

- $\quad$ the global knowledge which we can qualify knowledge as prior on the system. It is based on the past of the telecommunication system;

- the immediate knowledge, which corresponds to all the set of elements which are available at a given time to make a decision and operate. This knowledge is based on observations that can be digital or symbolic.

\section{Functional and dysfunctional modeling by graphs of states}

At the level of the various techniques, a first informal approach, based on intuition and experience, is necessary. However, it is not based on a methodology and quantified and validated data. Undetected side effects may occur.

A more systematic and formal study of the performances is therefore essential, by modelling then by observation of the behavior. If we only use observation, we may have to radically change a system that has already 
generated a lot of costs, because this technique is being used when the system has already been built.

\section{Knowledge model}

For telecommunication systems, the knowledge or functioning model is a formalization of its structure, in a natural or graphic language. The knowledge model, based on artificial intelligence, will contain all the knowledge acquired during the observation phases. This knowledge will be used to control or monitor the system, in order to anticipate the probability of a failure, and above all to avoid waiting times. For this, it is important to consider the action model

\section{Modeling Formalisms}

The formal modeling techniques that have been developed are based on the same principle in defining:

- the states of the telecommunication systems;

- transitions between states;

- transition delays.

The states are represented graphically by knots, and transitions, by labeled arrows connecting the knots between them (Tadepalli, 2008). The transitions are labeled by events whose occurrence causes a change in the state of the system. The resulting graph is known as a transitions diagram representing the behavior of the system.

The telecommunication system has a finite number of states. The change of state of the system is generated by the execution of an action that triggers a transition. But it is difficult to carry out a manual modeling of all the possible transitions between the different states of a large and complex telecommunication system. Often, the underlying transition diagram of a real system is of the order of a few thousand, even hundreds of thousands of states. Several high level formalisms ( Hillston, 1996) have been proposed to allow the modeling of a complex system through compact algebraic formulas and the automatic derivation at the lowest level of the transition diagram and the underlying Markov chain (Stewart, 2016). Each formalism has its own syntax. The derivation of the Markov chain is no more than an application of its semantics, generally defined in terms of transitions between states. A software is then used to generate the state space and the matrix « infinitesimal generator » of the Markov chain. It also makes it possible to calculate the stationary and transient solutions. Regardless of the formalism used, the goal is to calculate the performance parameters of the system.

Some methods of resolution are however specific to formalism, but technics can be adapted from one formalism to another. 
The Stochastic Automata Networks (Plateau, 1984) and (Fourneau, 1997), are a formalism of high level which allows the modeling of very large Markov chains and very complex, in a compact and structured way. They were built to take into account the size of the state space when designing a model. Indeed, a telecommunication system is modeled from several components often evolving in parallel (independently), except in certain synchronization actions where the components interact with one another. In this formalism, the behavior of a component of the system is represented by a set of states which constitutes an automaton (a Markov chain with a reasonable state space). Each automaton is described by a local matrix. The set of all the automata thus constituted is represented by a multidimensional Markov chain, whose states are those of the product space, with an underlying matrix called descriptor (Plateau, 1984). It is obtained by applying the operators of the tensor algebra (or of Kronecker) on the local matrices of each automaton. The generator structure allows a considerable gain of the memory space since it avoids the storage of the entire generator matrix. Effective resolution techniques (Durbach, 1998) and (Benoit, 2003) are then available for quantitative analysis. But, the use of independent components connected via the synchronization functions can produce a state space with many inaccessible states. In order to overcome all these limitations, we integrate the tools of Artificial Intelligence that are: Neural Networks and Fuzzy Logic.

\section{Preliminary interpretations}

In The dynamic capacity management of telecommunication systems remains a major challenge for telecom operators who must take into account the interest of Artificial Intelligence in the monitoring of these systems (Offole, 2016).

Any physical system is governed by two main dynamics which are: a continuous dynamic materialized by the differential equations and a discrete dynamics materialized by the Boolean equations (Perez, 2009).

In the case of a telecommunication system, these dynamics are defined as follows:

- the continuous dynamics $f$, characterized by the flow of message so the modeling was done by the first-order differential equations (1) of the form:

$$
\dot{X}=f(X, U, t)
$$

Where

$\dot{X}$ represents the variation of message flow in the system;

$U$ represents the input variables of the system;

$t$ represents the operating time. 
- the discrete dynamic, characterized by operating states of the main components of the system. It is modeled by the equations of the Boolean algebra.

The actual dynamic systems respond not only in function of time (continuous models), but also according to their environment and the events that may occur there. If the continuous models are good approximations of steady-state operation, and discrete models, good descriptors of the operating sequences of a system, it is clear that the global model closest to reality is a dynamic hybrid system.

Some events are random (component failure in particular) and confer a stochastic aspect of the problem. A model in the form of a hybrid stochastic automaton makes it possible to model the reality of a system control / command. Whatever the model used, standards and needs of safety and criticality requirements are strict for all command and control systems. The evaluation of system performance within a dynamic framework becomes a necessity.

A Hybrid Automaton (Perez, 2009) and (Marzat, 2008) appears as the association of finite state automata piloting a set of continuous dynamic equations. The equations modeling the continuous behavior of the system at a given instant therefore depend on the discrete state in which the system is found (Wolff, 1998). It latter can evolve according to the value of the continuous greatness.

A Hybrid Automaton is Stochastic, if the probability densities are taken into account associated with transitions between states, as well as clocks.

Formally, a Hybrid Stochastic Automaton is an 11-tuple:

$$
\left(X, E, A, \mathrm{X}, \mathrm{A}, H, F, p, x_{0}, \mathrm{x}_{0}, p_{0}\right)
$$

in which :

- $\quad X$ is a finite set of discrete states;

- $E$ is a finite set of events;

- $A$ is a finite set of arcs of the form (Figure 1) where:

$x$ et $x$ are the origin states and the arc, «e $»$ is the event associated with the arc, « $\mathrm{G} »$ the guard condition and « $\mathrm{R} »$ is the reset function. On occurrence of « $e$ » if the guard condition $« \mathrm{G} »$ is verified, the rocking of the state system $x$ to the state $x$, in which « $\mathrm{R} »$ defines the initial values of the continuous variables of the system;

- $\quad \mathrm{X}$ is a finite set of real variables; 
- $\quad \mathrm{A}: X \times X \rightarrow\left(R^{+} \rightarrow R\right)$ is a function of « activities », which associates to an element of $X \times X$ a defined function on $R^{+}$and has value in $R$;

- $\quad H$ is a finite set of clocks;

- $\quad F: H \rightarrow(R \rightarrow[0,1])$ is an application which associates with each clock, a distribution function;

- $\quad p$ is a state transition probability distribution $p\left(x^{\prime} x, e\right)$. For example, if we have the same event « $e$ » defining transitions from discrete state $x$ to the discrete states $x$ ' and $x$ " (it is said that there are conflicting transitions). We can define the probability $p$ to pass from the state $x$ to the state $x$ ' and the probability $(1-p)$ to pass from the state $x$ ' to the state $x$ ";

- $\quad x_{0}, x_{0}$ and $p_{0}$ correspond to the initial discrete state $x$, to the initial value of the continuous state variable $x$ and has the initial transition probability, respectively.

Once the Hybrid Stochastic Automaton model is obtained, a combination with neuro-fuzzy networks is necessary, to obtain the desired model, namely: « a neuro-fuzzy Hybrid Stochastic Automaton ». It will function to control the prediction error on telecommunication systems.

The modeling done takes into account the operation of the system without Artificial Intelligence on one hand, and on the other hand, by integrating Artificial Intelligence (a neuro-fuzzy ANFIS block).

\section{Method}

\section{S}

\section{Hybrid stochastic modeling of the telecommunication system}

The transmission of messages in a telecommunication system is carried out between the different stations (knots). These messages can borrow several paths to reach their recipients (stations).

Indeed, during this process, once the information is transmitted, the knots retrieve the reference or address of the recipient, through the intermediate of switch in the message header. Then, the router will multiplex the different messages on the determined output. During this process, the collision of information (or messages) can be recorded. They occur when receiving package. The small size of the bandwidth results in a significant loss of data. Hence the saturation of the network.

The reliability diagram characterizing the state model of the telecommunication system integrating failures (dysfunction), is modeled by a hybrid stochastic automaton (Fig. 2). His behavioral study is characterized by two main states: 
- the continuous state that takes into account continuous events (message flow, ...);

- $\quad$ the discrete state that takes discrete events into account (operating states of the various knots (stations)).

The various states in which a telecommunication system may be located as a function of transient events are:

State 1: The system is functioning normally; the data transmission is performed from one knot (station) to another.

State 2: The receiver knot has a failure. During this time, the transmitter is active and sends the information (or messages).

State 3: The receiver breaks down and goes into repair, while the transmitting knot (station) is always active and continues to transmit information to the receiver.

State 4: The transmitter break down in turn, while the receiver, after repair, becomes active again.

State 5: The transmitter is being repaired and the receiver remains active.

The model of states of such a system is shown in Fig. 2.

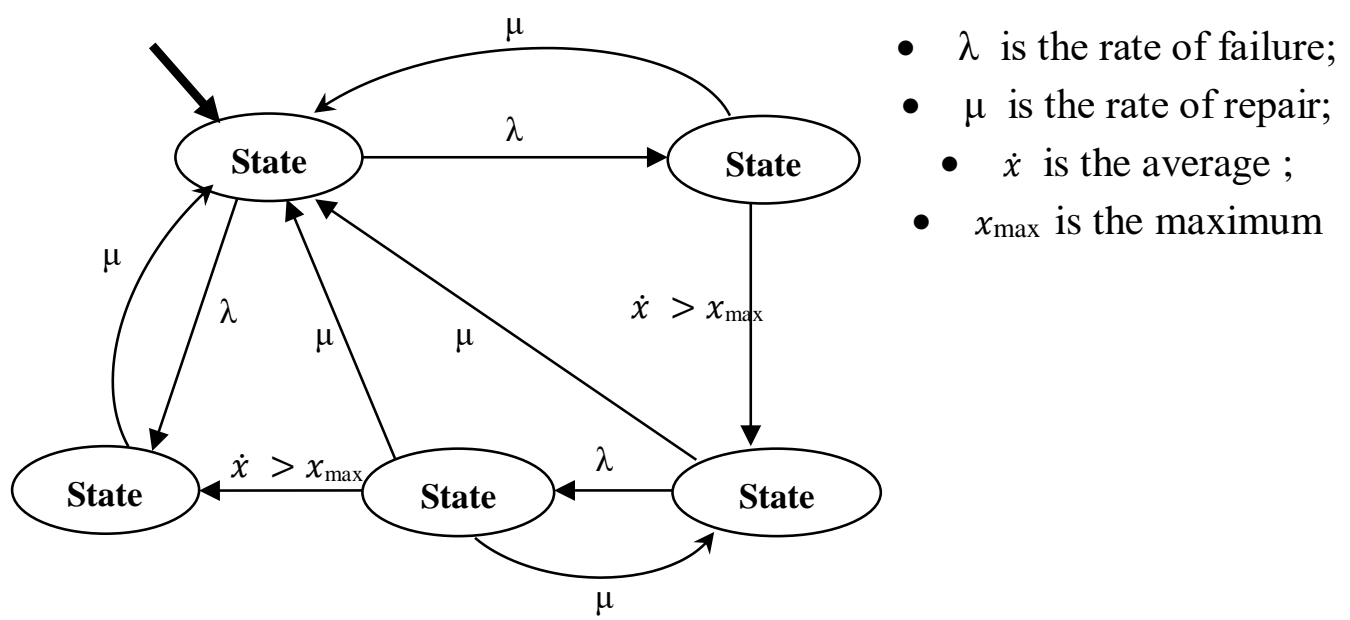

Fig.2: Hybrid stochastic automaton representing the state model of the telecommunication

The hybrid stochastic automaton model of Fig. 2 has been translated into Scilab whose model is given in Fig.3.

This scheme shows the mode block that represents the message flow as continuous variables used. It is constituted of different components namely :

- Mathematical expression : these are the mathematical equations. They define the continuous operation of the system;

- $\quad$ Extract : allows to extract the positive values of the variables; 
- Mux : is the adder, which allows to combine the mathematical expressions;

- $\quad$ The values 1, 2, 3: are the input ports, corresponding to the different variables of the system, such as : time, command, and state;

- A value of 0 is used to initialize the system.

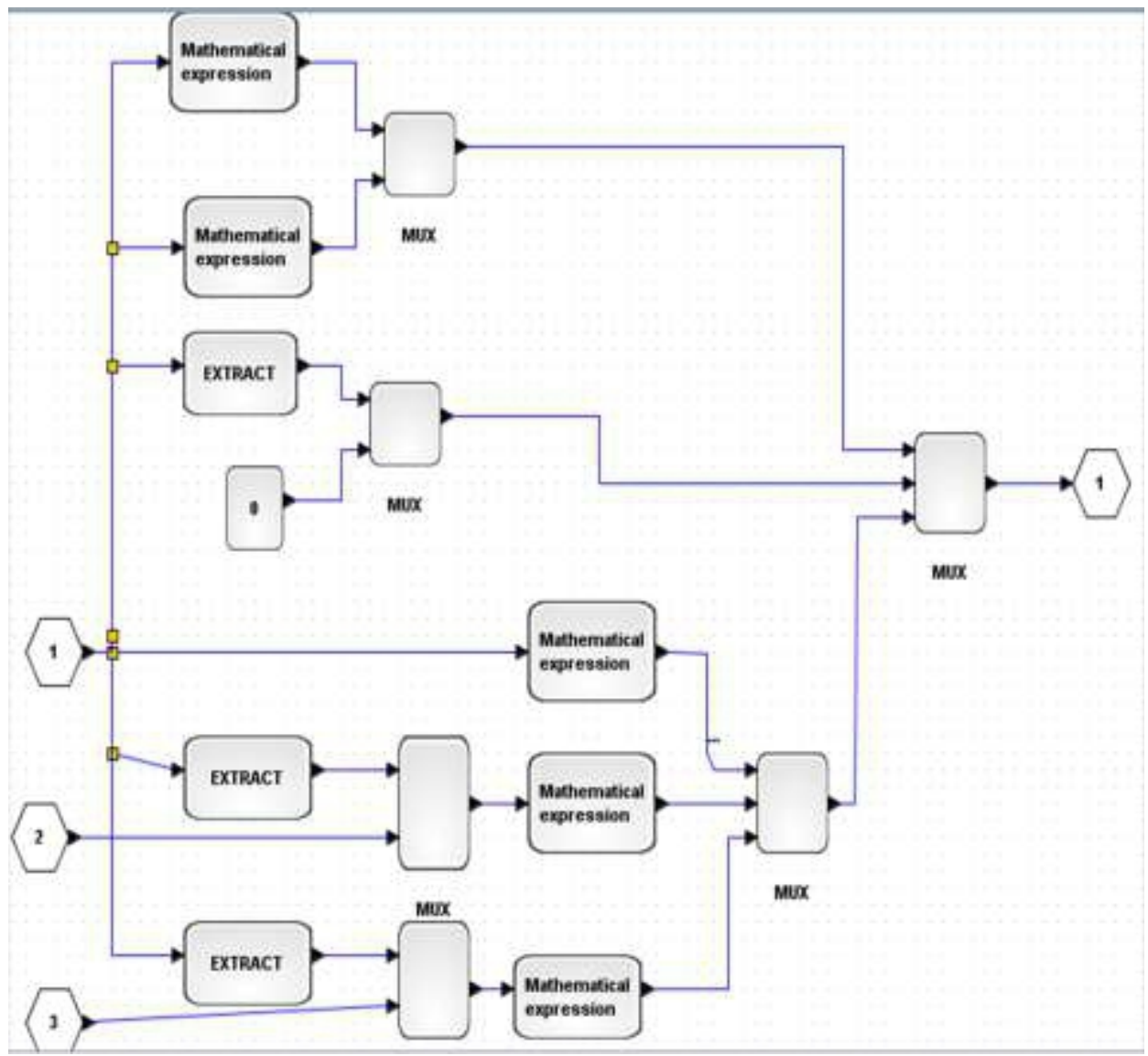

Fig.3: Mode block of the hybrid stochastic automaton

The Fig.4 shows the block of discrete variables, consisting of five modes. Each mode represents the five states of Fig.2. The continuous variable block of Fig. 3 is the exploded schema of a mode among the five modes of the discrete variable block of Fig.4. 


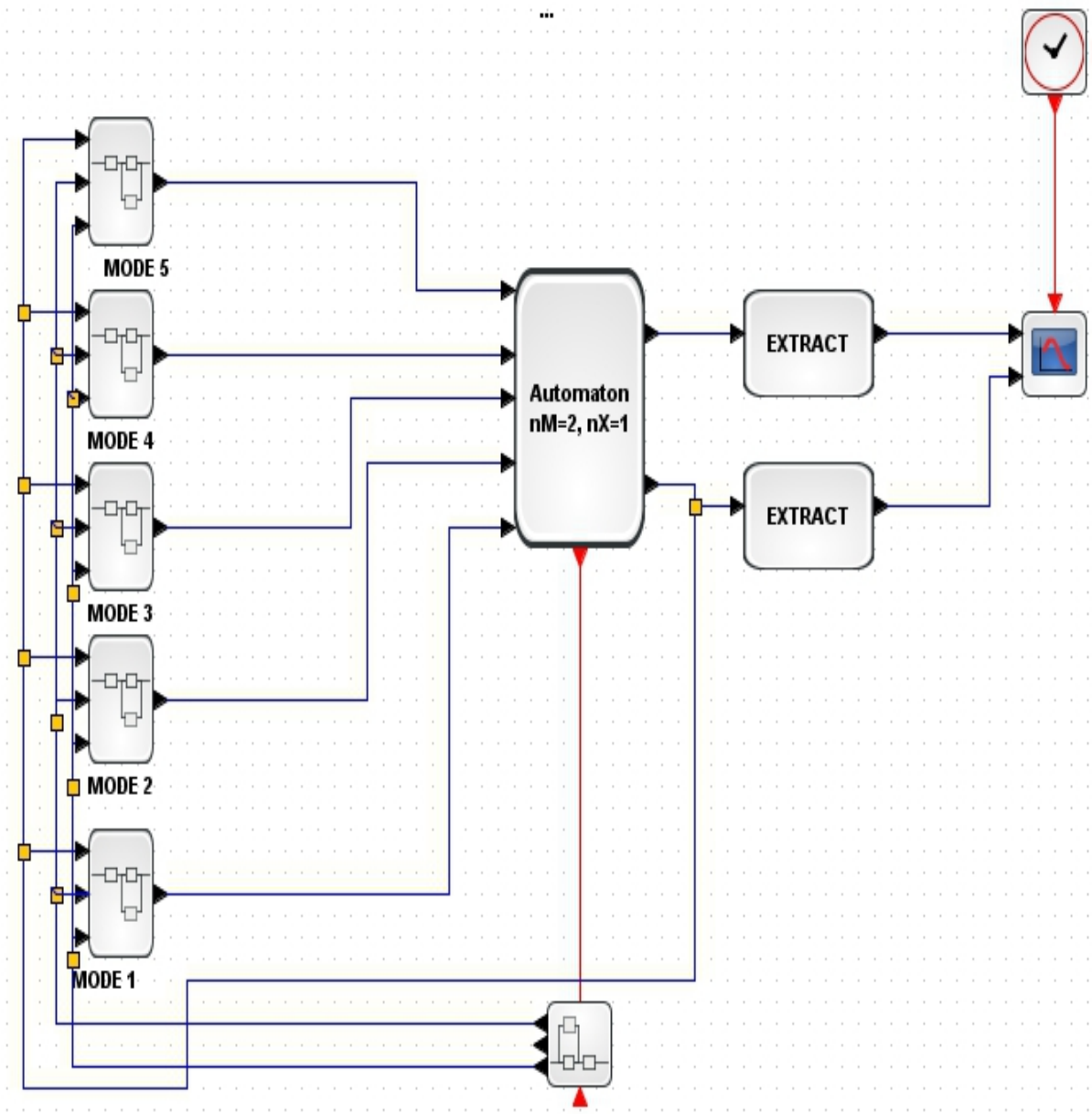

Fig.4: Generator block of the hybrid stochastic automaton

This scheme shows the generator block which represents the discrete states of the system. It symbolizes the operating states of the different knots (stations). It is constituted of different components that are:

- Mode: is the state of the system that corresponds to each of the five (05) states of the system shown in Fig.2;

- $\quad$ Extract: allows to extract the positive values of the variables;

- Automaton: this is the decision block, in which Artificial Intelligence (neuro-fuzzy networks) has been integrated.

\section{Functional Behavioral Study of the System Integrating Artificial Intelligence}

The objective of the behavioral study of the system is to better apprehend, control, appreciate and visualize, from the tools of Artificial Intelligence (Neuro-Fuzzy ANFIS networks) functional behaviors and 
dysfunctional of the telecommunication system, during its phase of useful life.

The aim is to anticipate the probability of occurrence of a possible failure, especially the progressive. The binary operation mode "on", "off" is prohibited. The system uses membership functions allowing an evolutionary control of an upstream nominal mode, to a downstream degraded mode.

Dynamic monitoring will be done favor to the ANFIS neuro-fuzzy networks (Fig 5). It allows to observe the presence of degradation, either to control its evolution. The expert (operator) can therefore be informed, allowing thus his intervention which would prevent lead a system failure (shutdown).

The modeling allows to know the functional and dysfunctional behaviors of the system. The indicators mainly target to check the accessibility of the service, its continuity, its availability and its reliability. They also affect the voices (failure rate, cutoff rate, success rate, ...) than on data transmissions (connection delay, download delay, reception rate, transmission delay, data error rate, ...), as well on the state of the system (safety functioning). They aim is to guarantee a satisfactory level of quality of service to customers.

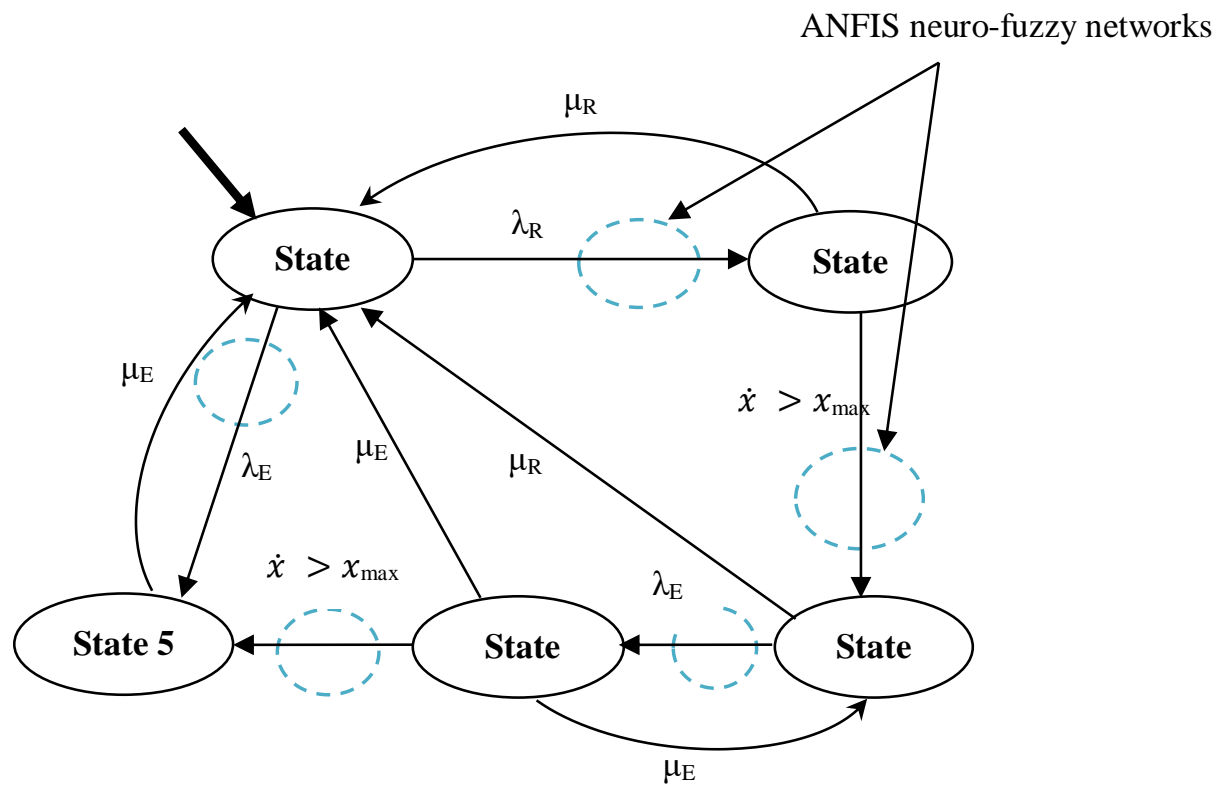

Fig.5: Hybrid Stochastic Automaton of a telecommunications system integrating artificial intelligence

The tools of Artificial Intelligence namely the ANFIS neuro-fuzzy networks have been integrated, to ensure decision-making at the transitions level between the nominal mode (on) and the degraded (off) mode. We represent the dynamics of the events inside the transitions. 
This modeling consists in replacing transition at a non-constant rate between two states, by a combination of transitions at constant rate (Cox model) (Kombe, 2011). It makes it possible to model any transitions, namely, the failures rates of increasing relating to a phenomenon of wear or deterioration. Thus, the Neuro-Fuzzy ANFIS networks are combined with transient states, to the control of the evolution of functional behaviors and system dysfunctional. This combination will allow make de good decisions, and to know the actions to lead for the smooth running of the system. The aim is to avoid that the system does not toggle in degraded mode. Integrated Artificial Intelligence makes it possible to fine-tune decision-making.

Whether the events are continuous or random, the expert can now notice the presence of degradation. Knowing that there is a class membership, there is a degree of confidence compared to the limits that the system will have to learn. The malfunction will be corrected thereafter thanks to the error gradient, which has the role of to master the error in the system.

\section{Results and Discussion of simulation of the dynamic system}

The softwares used for the simulation are: Scilab and Matlab/Simulink.

The simulation model of the telecommunication system used has been implemented under Scilab to obtain the generator blocks (Fig 3, Fig.4). Once the model is implemented on Scilab, we simulate on Matlab/Simulink to generate graphs of discrete events and continuous state.

This simulation of the model makes it possible to follow in parallel, the variation of discrete states (the operation of different knots (stations)) following discrete events, and which in turn impacting the variation of the message flow, from continuous events.

It also allows appreciating the operation of the various knots (stations) in the discrete state, without Artificial Intelligence and with Artificial Intelligence.

The Fig. 6 presents the variation of the discrete states (the functioning of different knots (stations)) following discrete events, and which in turn impacting the variation of the message flow from continuous events without Artificial Intelligence.

Fig. 6b shows the five (05) system states from discrete events. These events represent the functional states and dysfunctional of the telecommunication system. In Fig. 6a, we have to ordinate, the message flow and to abscissa the time (hours).

We considered that the maximum message flow is 40 messages, and the simulation time is 20000 hours, in the objective to better appreciate the behavior of the system. 
The system is at the beginning in the discrete state 1 when the time $t$ $=0$ until $\mathrm{t}=4800$ hours (Fig. $6 \mathrm{~b}$ ), which sending back to the nominal state. Then, the message flow is constant and stable at 40 messages (Fig. 6a). The data transmission is performed from a knot (station) to another. This situation obeys the defined functional behavior by the hybrid stochastic automaton of the system.

From $\mathrm{t}=5000$ hours to $\mathrm{t}=7100$ hours, the system state changes, that is to say passes from the discrete state 1 to the discrete state 2 . Then from state 2 to state 3 between 7100 and 11700 hours. This state change is due to the presence of a failure of the knot. This failure impacts the message flow when transmitting data (Fig. 6a). It is materialized by "peaks" very accentuated, which sending back to the state of dysfunction. Thereafter, the system switches from the discrete state 3 to the discrete state 1 between 11700 and 14000 hours, which bring back the system to the nominal state. Then, the system switches from the discrete state 1 to the discrete state 5 following a failure between 14000 and 14950 hours, because a knot is still under repair (Fig. 2). It therefore cannot have a data transmission, a less pronounced peak is observed at this instant (Fig. 6a). For the rest, the system switches from the discrete state 5 to the discrete state 4 between 14950 and 16200 hours. This state change is due to the presence of a failure of the knot. This failure affects the message flow when transmitting data. It is materialized by "peaks" very accentuated, which sending back to the state of dysfunction. From $t=16200$ hours to $t=17400$ hours, the system passes from the discrete state 4 to the discrete state 5 . This state change is due to the presence of a failure of the knot. This failure affects the message flow when transmitting data. It is materialized by a "peak" less accentuated, which sending back to the state of dysfunction. The system switches from the discrete state 5 to the discrete state 4 between 17400 and 18900 hours, following a failure of the knot. This failure impact the message flow when transmitting data. It is materialized by very "peaks" accentuated, which send back to the state of dysfunction. Finally, the system switches from discrete state 4 to discrete state 1 between 18900 and 20000 hours, which bring back the system to the nominal state. Transmission of the data stream becomes stable.

The control of the transition from "nominal mode" towards "degraded mode" is done without the integration of a tool of Artificial Intelligence. This is what can explain the very sudden tipping from a nominal state to a degraded state, without evolutionary transition from degradation, and without the expert to notice. This is observed at 14000 hours, where the system goes from state 1 to state 5 (Fig. 6a).

The curve of Fig. 7 shows the variation of discrete states (the operation of different knots (stations)) during time. The occurrence of 
discrete events impact in turn, the variation of the flow of messages (continuous events). This variation obeys the functional behavior and dysfunction defined by the hybrid stochastic automaton associated with neuro-fuzzy networks.

We considered that the maximum message flow is 40 messages, and the simulation time is 20000 hours. The system is initially in the discrete state 1 when the time is $t=0$ until $t=5400$ hours (Fig. 7b), which sending back to the nominal state. The message flow is constant and equal to 40 messages (Fig. 7a). The data transmission is carried out from one knot (station) to another. This variation obeys the defined functional behavior by the hybrid stochastic automaton of the system.

From $t=5400$ hours to $t=7200$ hours, the system switches from the discrete state 1 to the discrete state 2 . One observes then a fluctuation of the flow of messages, characterized by peaks, but this time, less accentuated (Fig. 7a).
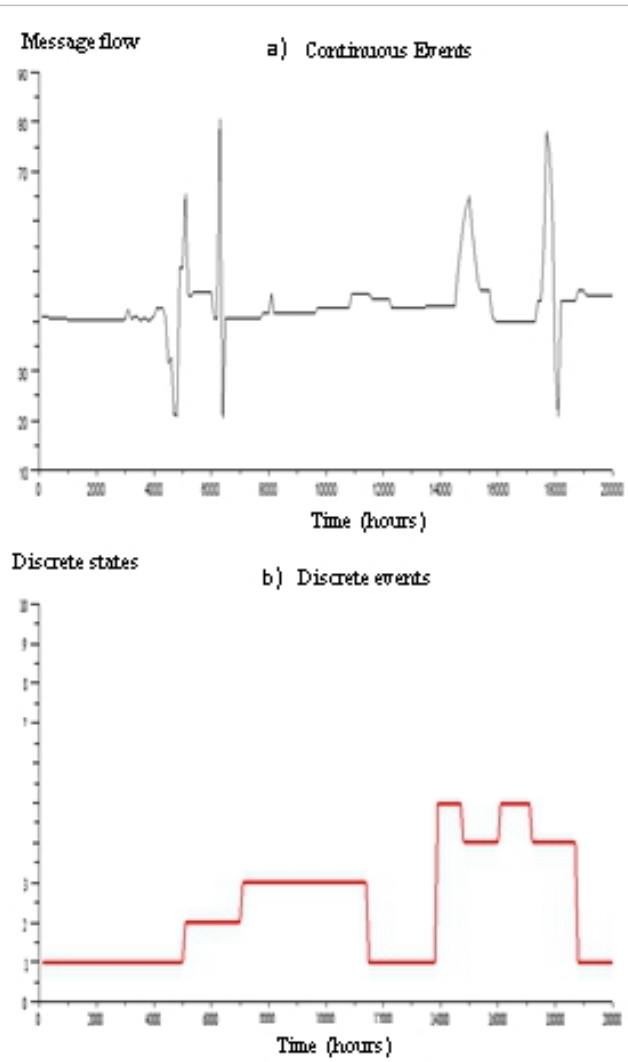

Fig 6: Simulation of continuous events and discrete with the hybrid stochastic automaton without attificial intelligence

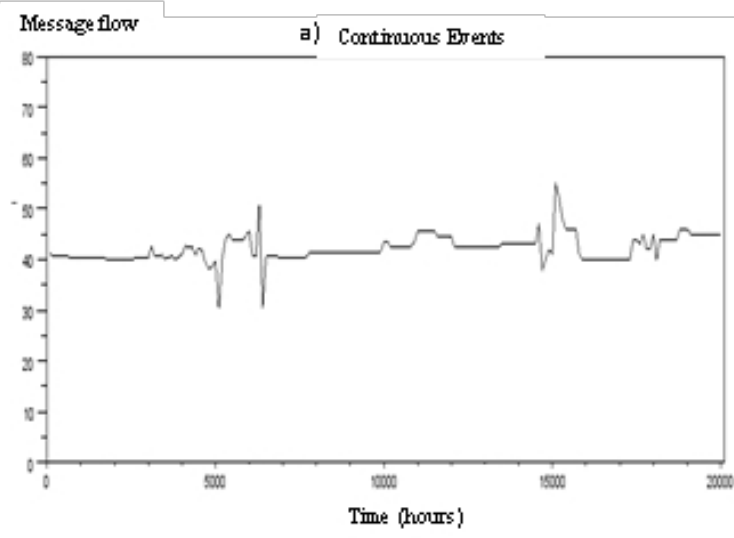

Discrete states

b) Discrete exterts

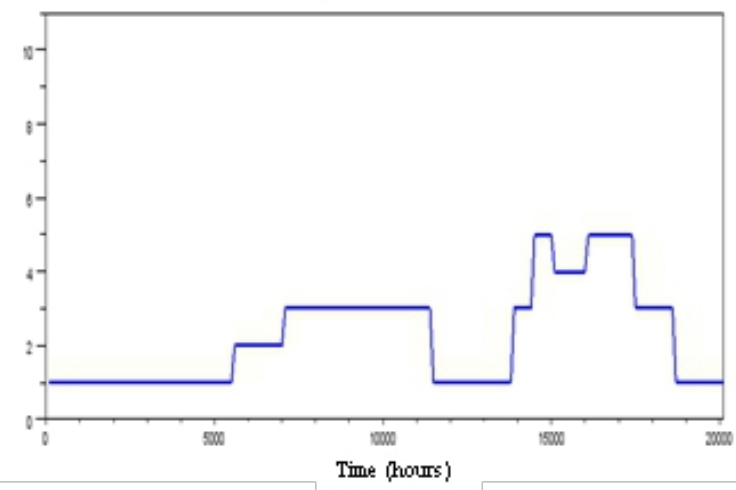

Fig.7: Simulation of continuous events and discrete with the hybrid stochastic automaton integrating artificial intelligence 
Thereafter, the system switches to the discrete state 2 to the discrete state 3 between 7200 and 11500 hours. This is due to the presence of a failure of the knot. This failure slightly impacts the message flow when transmitting data.

This decrease in amplitude of peaks observed on the curve is the result of the fact that a control of the gradual evolution of the degradation was carried out, which made it possible to anticipate on the dysfunction of the system. Thereafter, the system switches from the discrete state 3 to the discrete state 1 bringing back the system to the nominal operating mode.

The weak changes in states still observed do not impact not strongly the transmission of data, which oscillates slightly around 40 messages. In this model, the system works with the membership classes.

That is to say that the presence of a failure in the telecommunication system does not toggle this last automatically from nominal mode to degraded mode. This shift is fact rather of way gradual. This makes it possible to anticipate on the total failure, through to supervision exerted by the neuro-fuzzy networks.

\section{System Performance Indicators Integrating Artificial Intelligence}

The evaluation of some dynamic parameters namely: Reliability, Availability and Maintainability was made using the Hybrid Stochastic Automaton, combined with neuro-fuzzy networks ANFIS, implemented under Scilab. One simulates repeatedly the model designed for the determination of the parameters such as: the average duration of the running times between two consecutive failures of a repaired entity, or still called Mean Time Between Failure (MTBF), the average duration of operation of an entity before the first failure, or still Mean Time To Failure (MTTF), Mean Time To Repair (MTTR) integrating this time the Neuro-Fuzzy ANFIS networks.

The determination of all these parameters will allow to trace the evolution of reliability curves, Availability and Maintainability, undeniable performance indicators of a telecommunication system.

\section{Intelligent Reliability of the telecommunication system}

The Reliability of a system is the probability that it performs a required function under given conditions, during a given time $t$ (Villemeur, 1988).

Concerning the evaluation of the reliability of the system, we have limited ourselves to the evaluation of the MTBF. Subsequently, we calculate the failure rate $\lambda$ from the following equation (2):

$$
\lambda=\frac{1}{M T B F}
$$


Once $\lambda$ obtained, we calculate the reliability $R(t)$ (equation (3)). Recall that we are at the useful life stage of the system, therefore the events are characterized by the exponential law.

$$
R(t)=e^{-\lambda t}
$$

The simulation results show that the reliability curve is decreasing and almost damped. Over time, it tends to zero (0). We realize that initially, $\mathrm{A}(\mathrm{t})=1$ when $\mathrm{t}=0$. As time progresses, the system reliability curve begins to decrease but slightly.

This decrease of almost damped curve is due to the presence of neuro-fuzzy networks in the monitoring of the system. This variation shows the slow evolution of degradation systems with time, and the good performance of the system. The simulation duration here is $2 \times 10^{4}$ hours. Fig. 8 presents these results.

Reliability

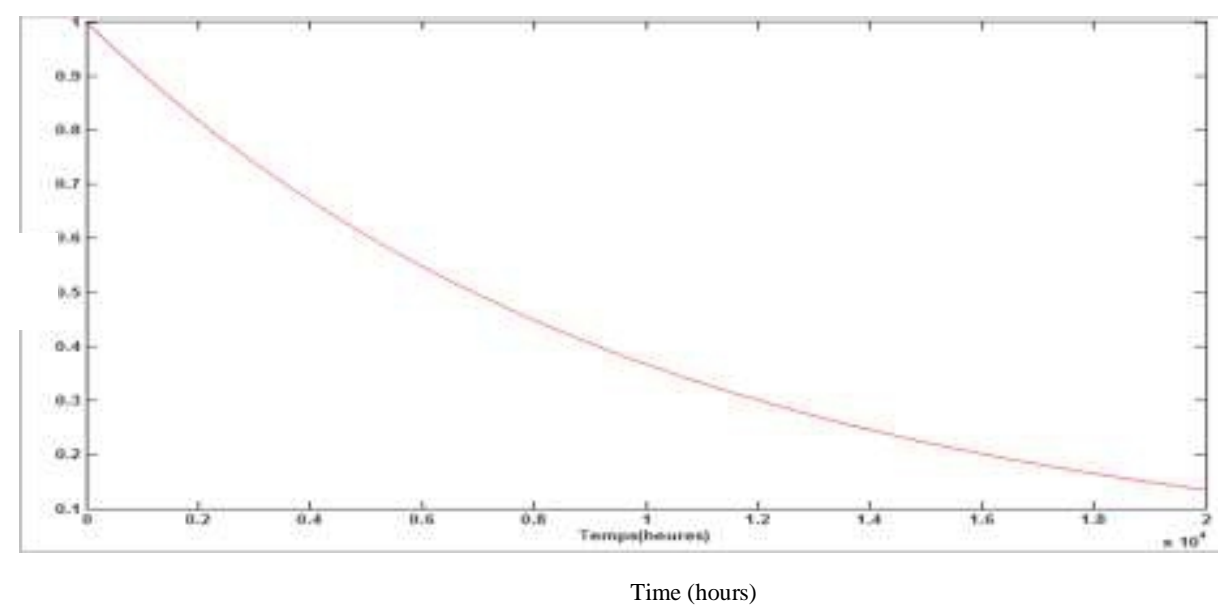

Fig.8: System reliability integrating neuro-fuzzy networks

\section{Intelligent Availability of the telecommunication system}

Availability is the probability that a system is in state to perform a required function under the conditions given at time $\mathrm{t}$ considered (Villemeur, 1988).

According to the curve of Fig.7a, the state of unavailability of the system is materialized by the presence of less "peaks" accented. These system failures do not necessarily render the system unavailable.

Concerning the evaluation of the Availability of the system, we first defined the MTTR. Subsequently, we calculate $\mu$ (the repair rate) from the following equation (4):

$$
\mu=\frac{1}{M T T R}
$$


Once $\mu$ obtained, we calculate Availability A (t) (equation (5)).

$$
\mathrm{A}(\mathrm{t})=\frac{\mu}{\mu+\lambda}-\frac{\lambda}{\mu+\lambda} e^{(\mu+\lambda) t}
$$

The results of the stochastic simulation of the state automaton show that Availability $\mathrm{A}(\mathrm{t})=1$ at $\mathrm{t}=0$. Over time, the curve decreases slightly and stabilizes at $85 \%$ (Value required by the World Class Performance) (Qmation, 2016) (Ayel, 2004) (Cimnet, 2004) and (Clemons, 2004).

This stability is due to the presence of neuro-fuzzy networks in the system. They already prevent errors due to failures. This shows that the system regains more stable availability. The simulation duration here is $2 \times$ $10^{4}$ hours. Fig. 9 presents these results.

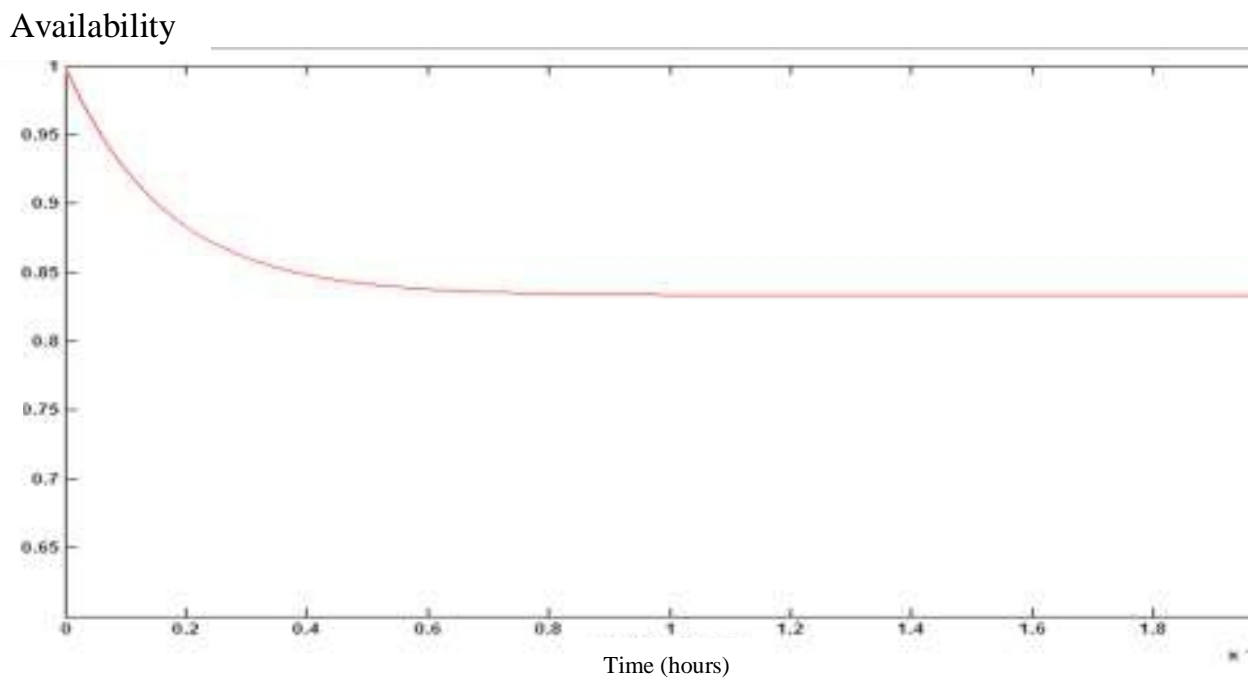

Fig.9: Availability of the system integrating the neuro-fuzzy networks

\section{Intelligent Maintainability of the telecommunications system}

The Maintainability of a system is the probability that the maintenance of an assured system under given conditions ends at time $t$, knowing that the entity was failing at $\mathrm{t}=0$ (Villemeur, 1988).

Once $\mu$ obtained by the formula (4), we calculate the Maintainability $\mathrm{M}(\mathrm{t})$ (équation (6)).

$$
M(t)=1-e^{-\mu t}
$$

The simulation results show that Maintainability is increasing and tends towards one. Initially, the Maintainability of the system is $M(t)=0$ when $\mathrm{t}=0$. Over time, the curve begins to grow slightly and stabilizes at 1 (Value Required for Optimal Maintainability). 
This growth is achieved thanks to the instantaneous reactivity of the tool of Artificial Intelligence which thus makes the duration of maintenance almost null. The simulation time here is $2 \times 10^{4}$ hours. Fig. 10 presents these results.

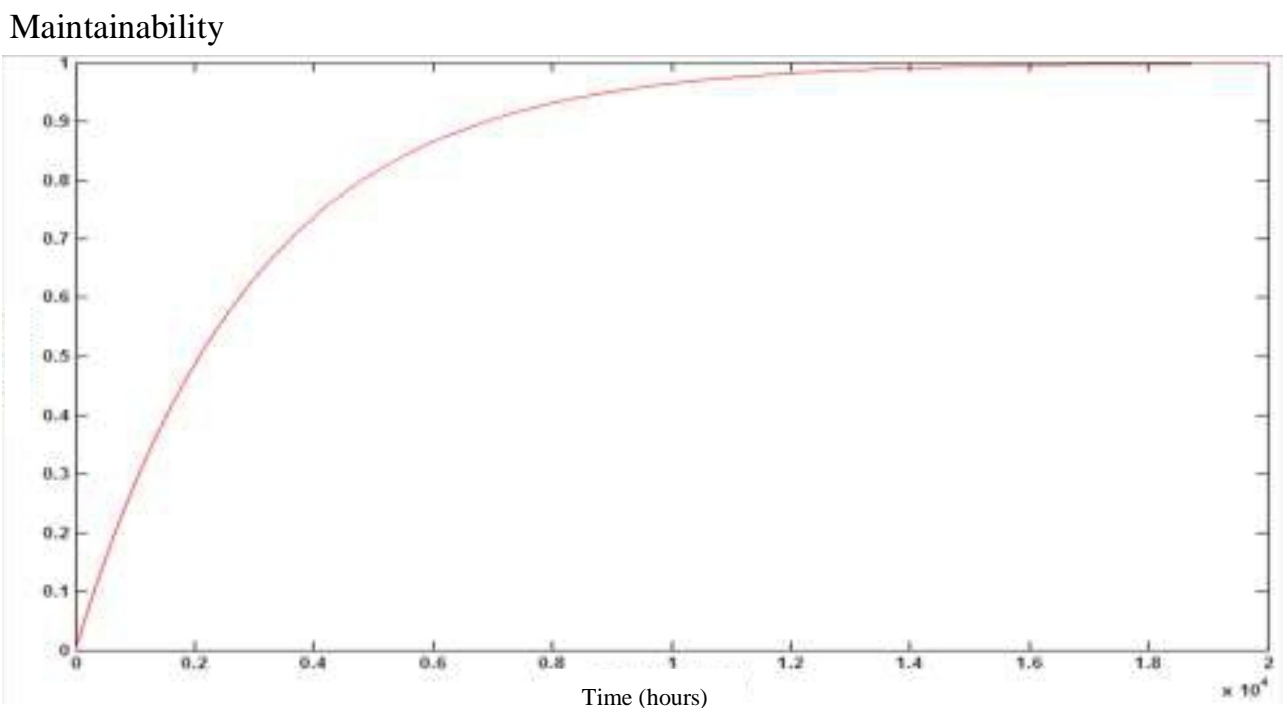

Fig.10: Maintainability system integrating neuro-fuzzy networks

\section{Intelligent Effectiveness of the Telecommunication System}

Define the performance of a telecommunications system is complex, because it groups several dimensions (Mathe, 1999). Starting from the two main assessment criteria of the performance that are: effectiveness and efficiency, a first classification of these dimensions can be realized. The success, competitiveness, the key success factors constitutes the main dimensions of appreciation effectiveness. The productivity, costs, yield and profitability, are those of appreciation efficiency.

The effectiveness is defined as the ability of an organization to achieve the objective it has set fixed (Jacot, 1997). According to (Longeaux, 1994), Effectiveness is the best possible report between the degree of customer satisfaction and the means implementation to obtain it. The customer satisfaction is in this case one of the dimensions of organizational success. The effectiveness, success are then close concepts.

We observe (Fig. 11) a very large difference between these two effectiveness curves. The effectiveness curve does not integrate the NeuroFuzzy ANFIS networks (blue) decreases rapidly with time and stabilizes at a lower $62 \%$ threshold.

This is due to the absence of control of the occurrence of failures in the system. What keeps it in a less high-performance state. The curve of the effectiveness integrating the Neuro-Fuzzy ANFIS networks (red) as for it 
presents a slight decrease and stabilizes at $67 \%$.

This shows that there has been anticipation on appearance of failures thanks to the mastery of the error of prediction. The effectiveness curve integrating neuro-fuzzy ANFIS networks is above the curve of the effectiveness not integrating the Neuro-Fuzzy ANFIS networks.

The Effectiveness curve is obtained from the combination of the three performance indicators previously evaluated namely: Reliability, Availability and Maintainability. Analysis of this curve allows to record that, at $70 \%$, effectiveness is average. It improves thereafter to reach a value close of $80 \%$ (threshold judged good). But this value could have reached $90 \%$ (threshold judged excellent) if one was making to pass the number of iterations to more than 46 .

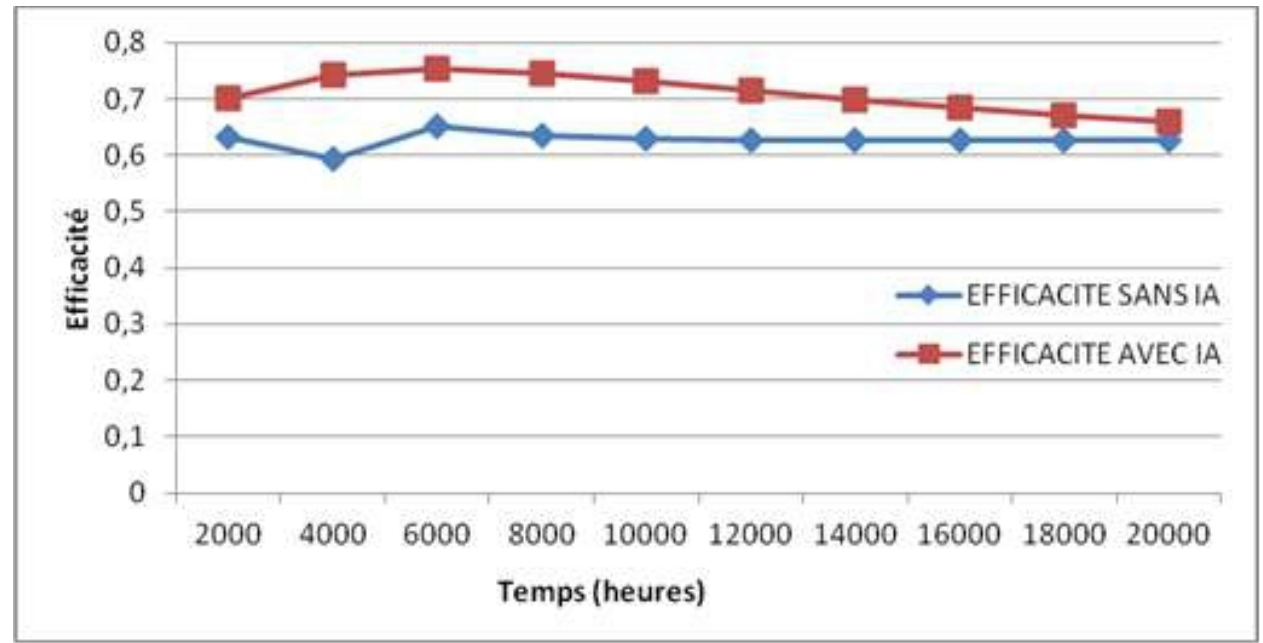

Fig.11: Effectiveness of the system integrating Artificial Intelligence (AI) neuro-fuzzy networks

\section{Conclusion}

We have shown the relevance of our approach by integration of dynamic Hybrid Stochastic Automata, for the performance evaluation of the telecommunication system.

It allowed us to combine discrete events (operating states of the different knot (station)) with continuous events (message flow) both from a deterministic and a stochastic point of view. Subsequently, the Artificial Intelligence tool namely the Neuro-Fuzzy networks has been integrated, in order to better diagnose and monitor by supervision with a smart block to the good going of the telecommunications system.

This integration of Artificial Intelligence has allowed to improved performance indicators that are: the Reliability, the Availability, the Maintainability, and the one of Efficiency who is effectiveness. This allows 
us to record that the systems of surveillance by Artificial Intelligence can be excellent decision support tools.

\section{References:}

1. Kombé, T., Niel, E., \& Rauzy, A. (2009). Modélisation Temporelle et Stochastique du TRS pour l'Evaluation de l'Efficience des systèmes de Production. $3^{\text {iemes }}$ journées Doctorales du GDR MACS (Groupement de Recherche en Modélisation, Analyse et Conduite des systèmes Dynamiques) JDMACS 2009, Angers-France.

2. Villemeur, A. (1988). Sûreté de fonctionnement des systèmes industriels. Edition Eyrolles, dans : Collection de la Direction des Etudes et Recherches d'Electricité de France (67 p).

3. Ruckus, W. (2009). Redéfinir l'économie du WLAN avec le maillage réseau intelligent. Ruckus Wireless | Livre blanc.

4. Ben Abdallah, A., Cheikhrouhou, O., Laurent-Maknavicius, M., Chaouchi, H., \& Ben Jemaa, M. (2006). Architecture de sécurité dans un réseau mesh multi sauts. 8th Symposium on Networks and Services GRES, Hammamet, Tunisia.

5. Lupan, R., Kobi, A., Robledo, C., Delamarre, A., \& Christofol, H. (2006). Modélisation et évaluation de la performance en conception. $6^{\mathrm{e}}$ Conférence Francophone de MOdélisation et SIMulation MOSIM'06 Modélisation, Optimisation et Simulation des Systèmes : Défis et Opportunités, Rabat, Maroc.

6. Benaicha, S., Mouss, H., Bencherif, F., \& Srairi, F. (2013). Commande Neuro-Floue d'un Système de Clinkérisation. International Conference on Electronics \& Oil: From Theory to Applications, Ouargla, Algeria.

7. Houacine, K. (2016). Commande neuro-floue d'une machine asynchrone dans une chaine de propulsion d'un véhicule électrique. Doctoral University mouloud mammeri of tizi-ouzou faculty of electric engineering and computing, Algerie.

8. Lefebvre, D. (2000). Contribution à la modélisation des systèmes dynamiques à événements discrets pour la commande et la surveillance. Habilitation to Lead researches University of FrancheComté/ IUT Belfort.

9. Tadepalli, S., Ramakrishnan, N., Mishra, B., Watson, T., \& Helm, F. (2008). Deriving Kripke Structures from Time Series Segmentation Results. Proceedings of the 9th International Workshop on Discrete Event Systems, Göteborg, Sweden.

10. Hillston, J. (1996). A compositional Approach to Performance Modelling. Doctoral Computer science, Cambridge University Press. 
11. Stewart, J. (2016). Introduction to the Numerical Solution of the Markov Chains. ([online], available from. http://lsmwww.epfl.ch/design_languages/Model_Sys_Mix/Document s/modelmix03.pdf [accesed 2016]).

12. Plateau, B. (1984). De l'evaluation du parallelisme et de la synchronisation. Doctoral Computing, University of Paris-Sud.

13. Fourneau, J. M. (1997). Réseaux d'automates stochastiques : Exemples et applications aux télécommunications. In Proceedings, Ecole d'informatique des systèmes parallèles et répartis, Toulouse, France.

14. Durbach, C., Quessette, F., \& Troubnikoff, A. (1998). Solving Large Markov Model Based on Stochastic Automata Networks. In Proceedings of the Advances in Computer and Information Sciences, Belek-Antalya, Turkey.

15. Benoit, A. (2003). Méthodes et algorithmes pour l'évaluation des performances des systèmes informatiques à grand espaces d'états. Doctoral INPG.

16. Offole, F., Kombé, T., \& Atangana, A. (2016). Formalisation et élaboration d'un cadre applicatif de surveillance dynamique des systèmes de télécommunication basée sur l'intelligence artificielle. Revue Internationale Afrique Science (pp326-338).

17. Perez, G. (2009). Evaluation par simulation de la sûreté de fonctionnement de systèmes en contexte dynamique hybride. Doctoral National Polytechnic Institute of Lorraine.

18. Marzat, J. (2008). Automate stochastique hybride et fiabilité dynamique. Project Graduation, ENSEM, 3A ISA-CPDS.

19. Wolff, R. (1998). Stochastic Modeling and the Theory of Queues. New Jersey, Prentice Hall.

20. Kombé, T. (2011). Modélisation de la propagation des fautes dans les systèmes de production. Doctoral INSA Lyon.

21. Q-mation. (2016). OEE-World Class Performance Reporting. A Division of Q-mation, Inc. [online], Available from: http://www.qmation.com/webpages/oee.htlm. .

22. Ayel, A. (2004). La mesure de performance des machines de production. CETIM : TRS performance.

23. Cimnet. (2004). Improve your Bottom Line Through Manufacturing CIMNET. Inc. web: www.cimnet.com.

24. Clemons, W. (2004). Overall Equipment Effectiveness, EnteGreat. Inc. Mountain Conference, Birmingham, Alabama.

25. Mathe, J. C. \& Chague, V. (1999). L'intention stratégique et les divers types de performance de l'entreprise. Revue Française de Gestion (pp 39-49). 
26. Jacot, J. H. (1997). De la trilogie : productivité, compétitivité, rentabilité à l'évaluation sociale de la performance industrielle. In BARRAUX, J., Entreprise et performance globale : outils, évaluation, pilotage, Commissariat Général du Plan, Paris, Economica (pp 29-38).

27. Longeaux, D. (1994). Contrôle de gestion : évolution depuis 30 ans et nouveaux défis. Problèmes économiques (pp18-21). 